\title{
AUTOMATIZATION OF CONTACT PRESSURE MEASUREMENT BETWEEN TRUNK ORTHOSIS AND PATIENT'S BODY USING A MATRIX TACTILE SENSOR
}

\author{
Radovan HUDÁK*, Viktória RAJŤÚKOVÁ*, Jozef ŽIVČÁK* \\ *Faculty of Mechanical Engineering, Department of Biomedical Engineering and Measurement, \\ Technical University of Košice, Letná 9,04200 Košice, Slovakia \\ radovan.hudak@tuke.sk; viktoria.rajtukova@tuke.sk; jozef.zivcak@tuke.sk
}

\begin{abstract}
Trunk orthosis affects trunk by a corrective-pressure mode with an objective to correct deformities (e.g. scoliosis). The effectiveness of this function depends on various factors, i.e. correct orthosis manufacture, correct application to patients and regular pressure corrective effect control. All these factors can be monitored by a system for measurement and evaluation of the contact pressure between the instrument and trunk in a real time and also by a so called "off line" system data recording. Presented article describes the application of made-to-measure sensor system TACTILUS (Sensor Products, USA) in different regimes (ambulance - A, home - $\mathrm{H}$, combined - HA and multi-sensory ambulance - MA regime), testing of chosen static and dynamic matrix tactile sensor (MTS) parameters and results of the testing application between orthosis and patients' trunks.
\end{abstract}

Key words: Matrix Tactile Sensor (MTS), Contact Pressure, Trunk Orthosis, Scoliosis

\section{INTRODUCTION}

The current state in the field of pressure measurement between orthosis and patient's trunk at spine deformities is presented by several studies that solve the issue only partialy.

Clin J. et al. (2010) in their study found out that compressive pressures do not exceed the value of $1 \mathrm{MPa}$ at the application of Charleston trunk orthosisThe same author, in his further study observed, while studying TLSO orthosis, that there is a frontal bending reduction by corset application with power of $60 \mathrm{~N}$ in $20 \%$ (Živčák et al., 2007). Wong M.S. et al. (2000) set the average pressure functioning by the means of force pad to body with values $7.09+/-1.77 \mathrm{kPa}(53.2+/-13.3 \mathrm{mmHg})$ while the fixation belts pass was $26.8+/-5,2 \mathrm{~N}$ (Penhaker et al., 2012). Pèriè et al. (2004) found out that sagittal reaction forces moved up to $47 \mathrm{~N}$ with the corset (Krištof and Hudák, 2010).

In 2006, Romano et al. (2006) measured contact pressure when applying corset within patients with idiopathic scoliosis. They applied a sensor with dimensions $7.62 \times 20.32$, thickness of $0.1016 \mathrm{~mm}$. While measuring the pressure in different positions of patient, they observed average pressure values moving from $264.3 \mathrm{~g} / \mathrm{cm}^{2}$ at sitting up to $431 \mathrm{~g} / \mathrm{cm}^{2}$ at exercising. Maximum values moved from the range of $367 \mathrm{~g} / \mathrm{cm}^{2}$ to $958 \mathrm{~g} / \mathrm{cm}^{2}$.

Based on the analysis of the studies carried out up to now, own sensor system was designed, following required dimensions, pressure range and the way of placing the tactile elements.

Suggested system for continuous contact pressure measurement between trunk orthosis and patient's body in a real time is a specific way of matrix tactile sensor (MTS) application in various trunk orthosis phases (manufacture, finalizing, application). Based on the four mentioned phases, four system regimes for dynamic pressure measurement were designed, i.e. ambulance $(A)$, home regime $(H)$, home - ambulance regime $(H A)$ and multi-sensory ambulance regime (MA).
The scheme of sensory system for contact pressure continuous measurement between force pad (trunk orthosis) and patient's trunk in a real time is described in Fig.1. Sensor fixation for dynamic measurement in a real time must be firm enough, however removable for the needs of the system application into a different force pad. Sensor thickness $(0.7 \mathrm{~mm})$ is considered within the trunk orthosis construction, mainly from the point of view of biomechanical force pad's function on the patient's body.

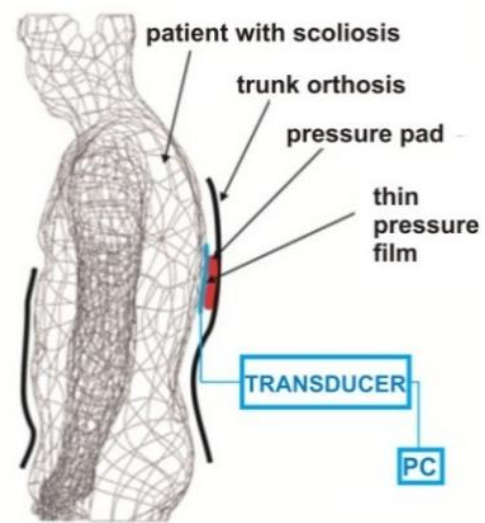

Fig. 1. Scheme of sensory system for continuous measurement in a real time

Real time measuring system, in the process of trunk orthosis manufacture can be applied at the control of shape and dimensions of the orthosis and way of placing the force pads. It can also be applied when trying the corset, before its finalizing, when the corset is applied directly on the patient with a possibility of further shape and biomechanical modifications based on the measurement outputs. The measurements will take place at the department of prosthetics and orthotics, both in phases of corset manu- 
facture and its testing, therefore it is an ambulance sensory system, i.e. A - regime (Fig. 2).

Measuring system is used also during the corset application phase. In this phase, biomechanical effect of the instrument is used from a long term point of view (patient wears corset in home environment). Biomechanical control was missing in this phase.

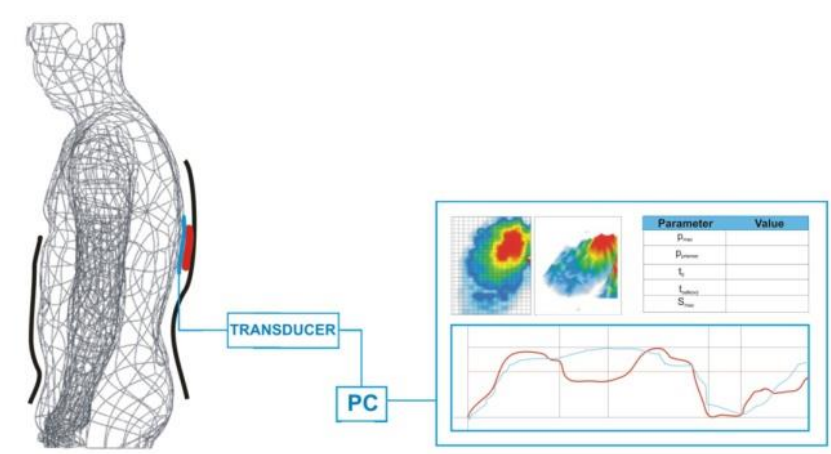

Fig. 2. Ambulance measure regime (real-time)

Therapeutical effect of the corset was monitored on the basis of regular scanning considering the invasiveness of RTG or CT diagnostics.

The measuring system was named as home (off-line) or so called $\mathrm{H}$ - regime (Fig. 3), where the pressure effectiveness scanning is carried out in home environment, but the analysis and evaluation is done in the orthopedic ambulance after the data is downloaded. The data transfer in home regime is possible by a cable (Fig. 3-1) or by wireless transfer (Fig. 3-2).

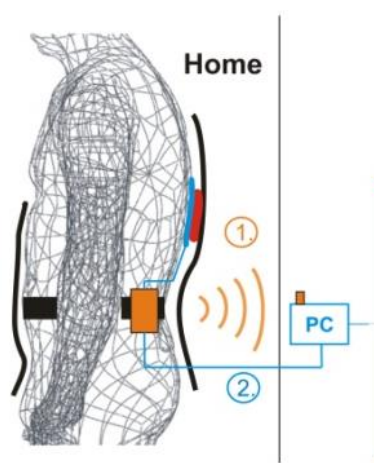

Ambulance

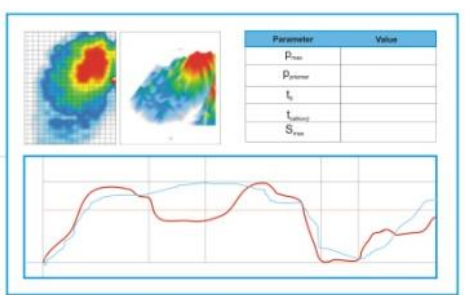

Fig. 3. Home regime (off-line), so called $\mathrm{H}$ - regime of pressure scanning

In the corset application phase, so called $\mathrm{HA}$ regime can also be used - it means home and ambulance regime (Fig. 4), where the data can be transferred in a real time. Thus, orthopedist can monitor the situation and effect of trunk orthosis application in any place and intervene if necessary. Home - ambulance regime measurement can have different ways of data transfer (Fig. 4. PC - $\mathrm{H}$ - home computer, $\mathrm{PC}-\mathrm{A}$ - computer in ambulance).

In the Fig. 5 there is a multi-sensory pressure scanning system between trunk and various force pads at the same time in a real time. This system enables to analyze pressure modes between more force pads and thus, draw a complex biomechanical trunk orthosis effects on patient. Mentioned system requires a solution of multichannel sensory system with a specific data collection point and own software application in order to synchronize pressure outputs from the individual force pads.
Outputs, analyzed using software application are evaluated qualitatively (2D and $3 D$ colour maps), and also quantitatively, where the situation is evaluated using particular pressure and time data and statistically processed values. For the purposes of MTS system made-to-measure testing, an ambulance measurement regime (A-regime) was chosen, used for testing the orthosis manufacture effectiveness.

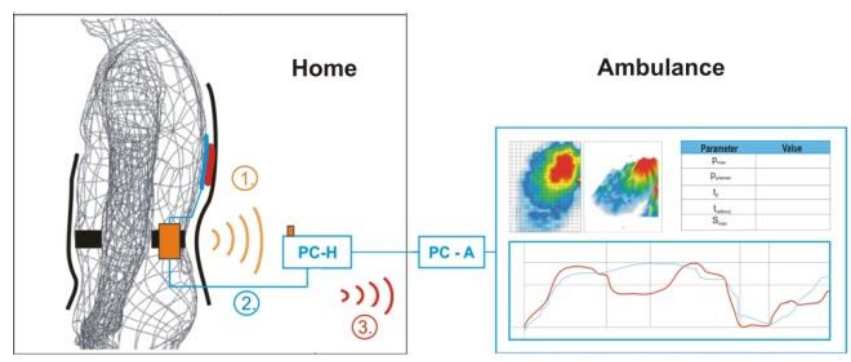

Fig. 4. HA - Home (off-line) - ambulance (real - time) regime measurement

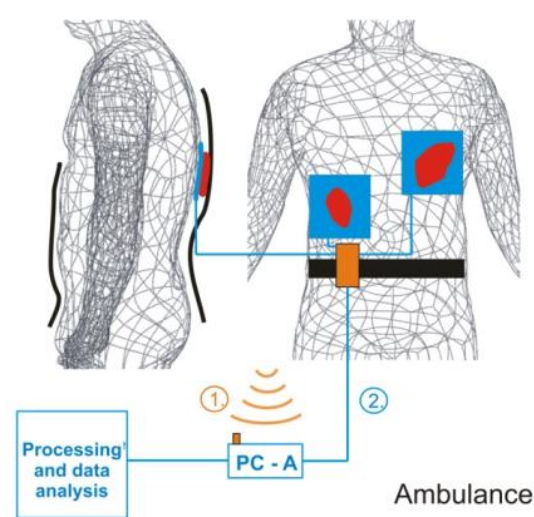

Fig. 5. MA - Multi-sensory ambulance regime measurement

\section{AMBULANCE MEASUREMENT SYSTEM (A-REGIME) - MATERIAL AND METHODS}

Ambulance measurement system in a real time consists of measurement pressure mat application between force pad and patient's trunk in ambulance, considering that the process requires continuous professional assistance at the time of measurement. The objective of the ambulance system is to verify the corset construction (shape, dimensions) before delivering it to a patient. At the same time, at regular visits in ambulance, it is possible to control the effect of force pad from the biomechanical point of view and thus verify any need of corset or force pad modification in order to optimize the pressure effect.

System function verification was carried out from so called A regime of scanning, in the phase of corset manufacture. In this phase, it is possible to measure and analyze the shape and dimensions of corset construction and verify the way of placing of force pads based on their pressure effect. On the basis of studies, a sensor system was designed and manufactured in order to measure contact pressure between trunk and orthosis force plate in a real time. The sensor system consists of a sensory mat $(20 \times 20 \mathrm{~cm})$, signal converter and connecting cables. The pressure range MTS was set from 0 to 724 PSI (from 0 to 729 $\mathrm{mmHg}$ ). In order to ensure free movement of the patient with 
applied pressure mat, suggested length of the connecting cable was 10 meters. Wide cables with 40 veins represent signals from the individual sensors which create matrix of 400 scan points. After the system hardware modules were set up, work with the software application TACTILUS (Sensor Products, USA) started. This application serves not only for processing and evaluation the measured data, but also for operating the whole sensor system. After the final record editing, the software is able to generate a text file (.txt) which contains all recorded images. The output file contains information about pressure values, maximal, minimal, average pressure and final force out of the matrix. In order to verify the system and process the methodology of measurement for ambulance system, testing measurements were carried out to find chosen static and dynamic MTS parameters and measurement in 10 subjects. The aim of the measurement was to find the entry parameters for MTS applications to the place of scanning, find the MTS time response and other metrological parameters and also to design optimal measurement methodology in ambulance regime. The aim was also to find pressure ratios and define referential graphs of pressure-time $(p-t)$ and surface - time (S-t) dependences for the exercises defined above.

It was needed to carry out the testing of the chosen parameters in order to verify the set entry measurement methodology of biomechanical effect of force pad to trunk of the measured subject. The measurements were not aimed to test the measurement accuracy, but to find stability of measurement and of chosen static and dynamic parameters. Testing was implemented by $2 \mathrm{~kg}$ weights placed to the centre of the pressure mat.

Considering the fact that the aim of the measurement was measurement system verification for the purposes of processing the measurement methodology of the contact pressures between force pad and trunk, where relative changes are important, there was no temperature influence to measurement process analyzed, neither influence of the mat to output data was analyzed. It was identified by testing that hardness; shape and material of the mat have a significant influence on the measured data accuracy.

Methodology processing requires considering more factors, parameters and secondary measurements which will be considered at processing and evaluating the results. At measuring, it is necessary to consider parameters of the subjects (S01: S10), environmental parameters (temperature, humidity) and their changes in the course of measurement, corset shape, force pad placing and pressure mat fixation. Other important facts are the hardware set up, choice and correct application of defined exercises, software application and input parameters set up, data processing, numerical and graphical presentation of the results, evaluation of the results and their interpretation to the engineering and clinical practice.

The measurement took place in a special laboratory with dimensions of $6.2 \times 3.5 \mathrm{~m}$. The temperatures shifted during all measurements between $21.5-24.4{ }^{\circ} \mathrm{C}$ and relative humidity between 32.1 - 49.5\%. Mentioned conditions met the requirements of measurements from the point of view of measuring system and thermal satisfaction of the measured objects.

Two types of corsets were applied to the measured subjects (S01 : S10) in a way that pressure between force pad and subject's body was created. Compression force between the force pad and mat was satisfactory for fixing the mat on the required place without moving, that is the reason why the pressure mat was not fixed.

From the point of view of placing of force pads, physical parameters of the objects and required pressure effects, there were
3 force pads (P1, P2, P3) used on the first corset (K1) and 2 force pads (P1, P2) on the second corset (K2).

After the correct pressure mat application, set procedures and exercises followed and were supported by biomechanical purpose or the disease itself (scoliosis).

Exercises and movements were set on the basis of available studies in the field of biomechanical analysis of corset influence to patient's trunk. The movements were applied to a control group of subjects with the aim of finding out the pressure ratios at individual exercises and set exemplary pressure procedures and size of surface in time. For all the force pads, movements like standing, forward bend, kyphotisation, kyphotisation on knees, backing out and raise arms. Using force pads, placed on the back or on the side of the corset, exercises like sitting, sitting and leaning, outstretching, lying on the back were preformed.

The setting of the exercises is followed by setting of the software application for contact pressure measurement in corsets and processing of the measured data.

It is crucial to set the parameters in the software application before scanning the pressure placement in order to obtain feedback about the correct pressure mat application, starting the measurement and satisfactory signal for a successful recording.

In order to visualize the placing of force pad's shape it was necessary to use interpolation functions. TACTILUS software offers some of the types, such as interpolations for pressure map editing, inverse distance weighting method (IDW), spline abilinear interpolation. However, in order to ensure correct result evaluation, it was necessary to use other types of interpolations, too.

After the correct entry data set up, recording of the measurement followed. With every subject (S01 : S10), every movement or exercise and every force pad, a video record of surface pressure placing was made in contact areas between force pad and pressure mat or trunk orthosis and pressure mat (Fig. 6).

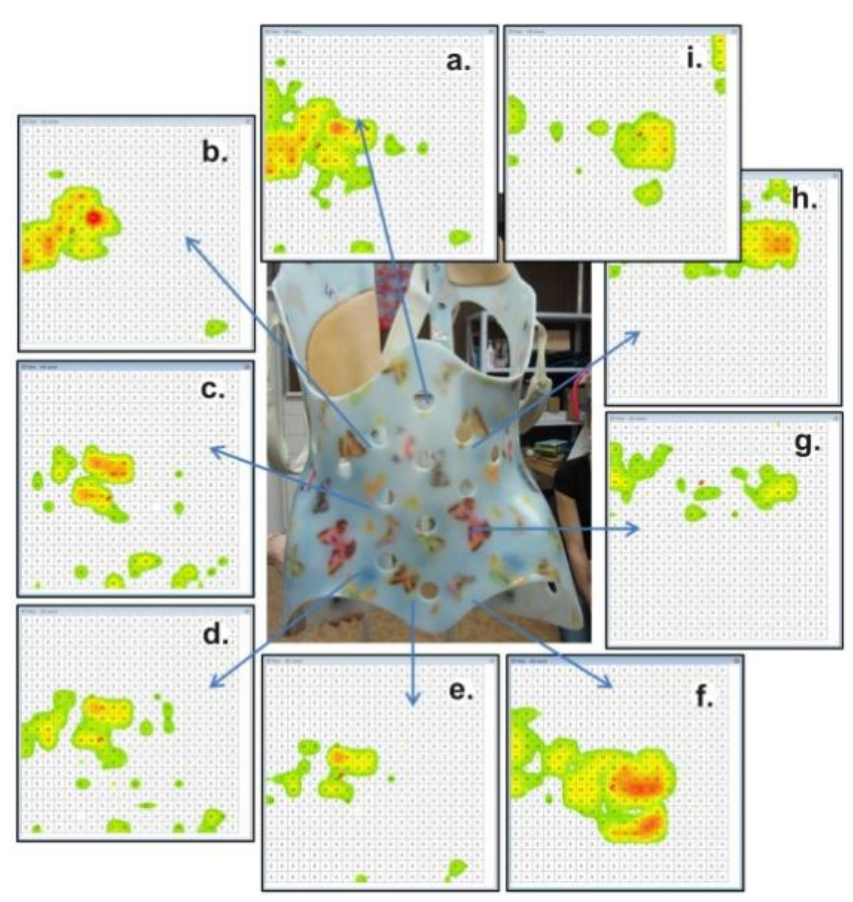

Fig. 6. $2 \mathrm{D}$ image with a maximum pressure value at various exercises and movements: a) kyphotisation, b) kyphotisation on knees, c) standing, d) backing out, e) raise arms, f) forward bend, g) sitting, h) sitting and leaning 
The software application enables to export the files in a text format (.txt) as well as export the whole pressure data matrix. Except numerical data, it is possible to export graphs of pressure and surface processes in time. The software also covers functions for generating force "F" dependence on time $t(F-t)$ which uses average pressure $\left(p_{\text {avg }}\right)$ for the calculation (Fig. 7).

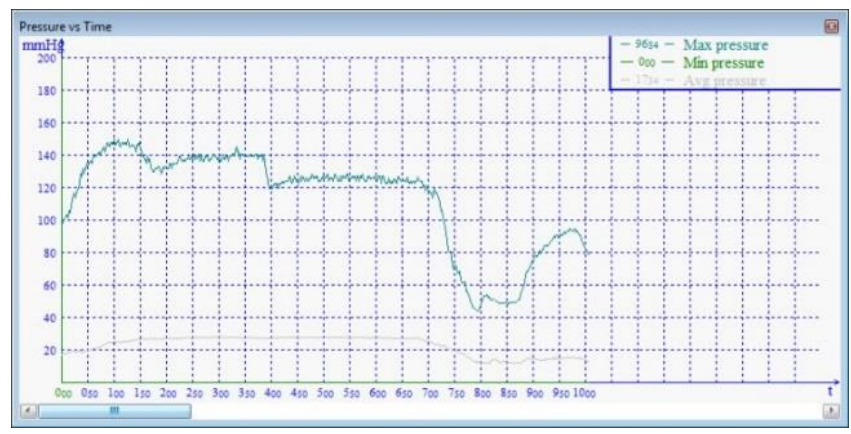

Fig. 7. Functionality graph: pressure-time $(p-t)$ dependence for maximal $p_{\max }$, average $p_{\text {avg }}$ and minimal $p_{\min }$ recorded values

\section{RESULTS OF SENSOR SYSTEM TESTING AND INTRODUCTORY MEASUREMENTS IN AMBULANCE}

Before measuring the pressure between force pads and trunk of the tested subjects, it was necessary to carry out testing measurements in order to verify pressure mat and the whole sensor system functionality which enabled to set the methodology directly on the objects of measurement more effectively.

\subsection{Testing results of chosen parameters of the pressure mat}

There were short testing measurements effectuated on the pressure mat in order to obtain basic static, dynamic and noise properties. Series of repeated pressure measurements were taken caused by weights placed in the centre of the grid, where there is supposed area for placing force pads. These simple measurements enabled to find the response for step pressure change, estimate standard repeated measurement uncertainty and consider the short-term noise parameters of the device.

The first measurement series were aimed to determine the measuring system response, while its sensory system is under step pressure change. It is possible to characterize manually the typical response process from the zero to the second stable position (placing the weights on a sensory grid) or to characterize the transfer to a stable position determined by the size of weight and effective contact surface.

By these characteristics, the introductory contact pressure measurement phase is monitored, between force pad and trunk, where the pressure mat is placed and demonstrates the beginning of the scanning process where there is step pressure change due to the application of weights.

Based on the analysis of the mentioned processes it is clear that dynamic properties of the scanned sensory matrix are well approximated for the contact area evaluation by the following formula:

$S=S_{\text {max }}\left(1-e^{-t / \mathrm{T}}\right) \quad \mathrm{T} \approx 0,8 S$ where the time constant of transfer from the second stable status is approximately 0,8 seconds. The overall contact surface is calculated on the basis of sensory grid pressure signals integration, thus it will correspond to the dynamics of the grid sensors.

Repeated measurement series were carried out with weights of 1 and $2 \mathrm{~kg}$ in 30 second time intervals at sampling frequency $49 \mathrm{~Hz}$ considering that the position of the weight was changed at every measurement. The aim was to qualify the repeated pressure measurement accuracy that depends on electrical, material, geometrical and constructional parameters of piezoelectric sensors' network.

For every series, there were basic statistic surface properties calculated (arithmetic mean and median) and width (diffusion and standard deviation) of the data division and set type A uncertainty. The following relations were used:

$\bar{p}=\frac{1}{n} \sum_{i=1}^{n} p_{i} \quad s^{2}(p)=\frac{1}{n} \sum_{i=1}^{n}\left(p_{i}-\bar{p}\right)^{2}$

Standard uncertainty of every measurement series was subsequently calculated based on the relation:

$u_{A}(\bar{p})=s(\bar{p})=\frac{s(p)}{\sqrt{n}}=\sqrt{\frac{1}{n(n-1)} \sum_{i=1}^{n}\left(p_{i}-\bar{p}\right)^{2}}$

Based on the calculated statistical properties, it is possible to state that the overall arithmetic pressure mean out of all series carried out by repetitive measurement is $\bar{p}=43.25 \mathrm{mmHg}$ a standard deviation $s(p)=0.92 \mathrm{mmHg}$ and the standard uncertainty type $\mathrm{A} u A(p)=0.07 \mathrm{mmHg}$.

For the B type standard uncertainty, producer data were used. The error $10 \%$ of the measuring system represents value of $4.32 \mathrm{mmHg}$ out of all the repeated measurements series. As we suppose equal division of the error probability of the measuring system, the B uncertainty can be expressed as follows:

$u_{B}(p)=\frac{4,325}{\sqrt{3}} \approx 2.50 \mathrm{mmHg}$

Overall measuring system uncertainty:

$u_{C}(p)=\sqrt{u_{A}(p)^{2}+u_{B}(p)^{2}}$

After substitution, the overall measuring uncertainty is $u_{c}(p)=2.50 \mathrm{mmHg}$ and the determining factor is the measuring system accuracy.

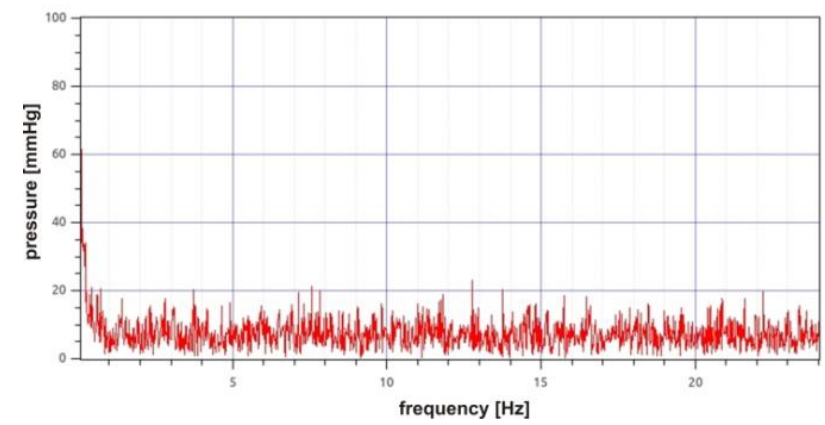

Fig. 8. Functionality graph: pressure-time $(p-t)$ dependence for maximal $p_{\text {max }}$, average $p_{\text {avg }}$ and minimal $p_{\min }$ recorded values

From the point of view of sensitivity and differentiating ability of the measuring system, knowledge of noise properties is crucial. It is important to consider the noise also when the interference 
can influence the information in noise or when the information itself is "drowned" in the noise and it necessary to acquire and reconstruct it.

Spectral noise properties are relatively constant in time and it is possible to derive noise capacity out of them that is concentrated in a particular frequency zone. In Fig. 8 there is an amplitude frequency spectrum of typical noise signal and it is clear that it does not contain any significant spectrum of line, it is flat and it responds to thermal noise spectrum.

Statistical properties are constant and noise values "very distant" from zero are little probable. Based on these facts it is possible to state that the noise in the signal does not influence the amount of the measured constant.

\subsection{Results of the introductory measurements using TACTILUS system in ambulance A-regime}

The results of introductory measurements were processed to charts and graphs for every movement and exercise. Values of the average contact pressure $p_{\text {avg }}$ and contact surface $S_{\text {avg }}$ and also maximal contact pressure $p_{\max }$, and contact surface $S_{\text {max }}$, were obtained.

Kyphotisation is presented by maximal contact pressure (Fig. 9) in the range from 0 to $40 \mathrm{mmHg}$.

Maximum contact pressure $p_{\max }$ during kyphosis

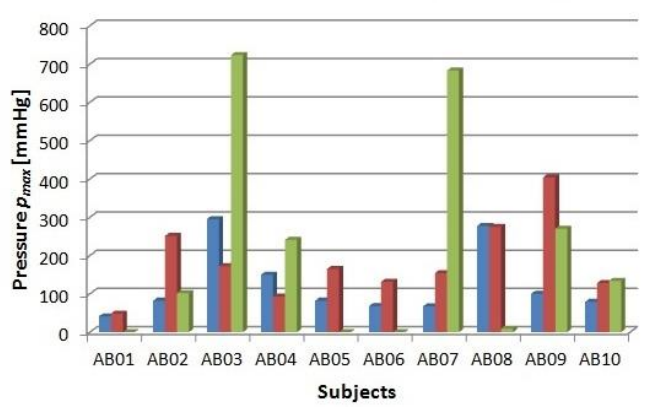

Fig. 9. Maximal contact pressure at kyphotisation within individual objects of measurement

Based on the graphic data presentation (Fig. 10) it is clear that the largest contact surface is at the force pad P2 and the values range from 0 to $100 \mathrm{~cm}^{2}$. The lowest values are at the force pad $\mathrm{P} 3$ and they range from 0 to $40 \mathrm{~cm}^{2}$.

Based on the analysis, it was stated that force pad P3 show higher average and maximal contact pressures, so do force pads $\mathrm{P} 1$ and $\mathrm{P} 2$, the majority of average contact pressure values range from 0 up to $200 \mathrm{mmHg}(0-26,6645 \mathrm{kPa})$.

The lowest values of average and maximal contact pressure are to be found at raise arms. On the other hand, it is not possible to clearly define during which exercise there are the highest values of average and maximal contact pressure; on the whole, the values are higher at kyphotisation and kyphotisation on knees. Within the average and maximal contact surface it is not clearly defined which exercise represents the highest or the lowest values.

Graphs in Fig. 11 and 12 show maximal pressure $p_{\max }$ and maximal surface $S_{\text {max }}$ at individual movements on force pad P2.

At the force pad P2, specific movements and exercises were carried out and they influence of force pad placed at the back part of the trunk orthosis. The highest pressure at force pad P2 was monitored while lying on the back, the lowest while outstreching.

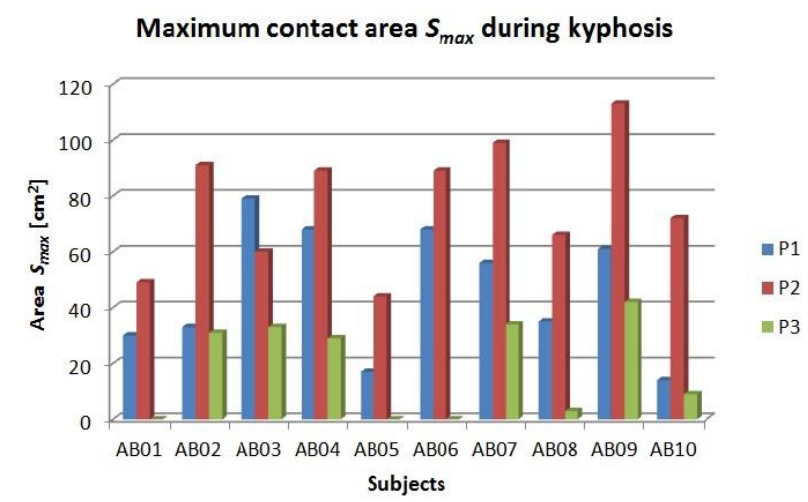

Fig. 10. Maximal contact surfaces at kyphotisation within individual objects of measurement

Pressure variations directing upwards or downwards within the individual objects of measurement describe various physiological proportions of the objects of measurement (various BMI index).

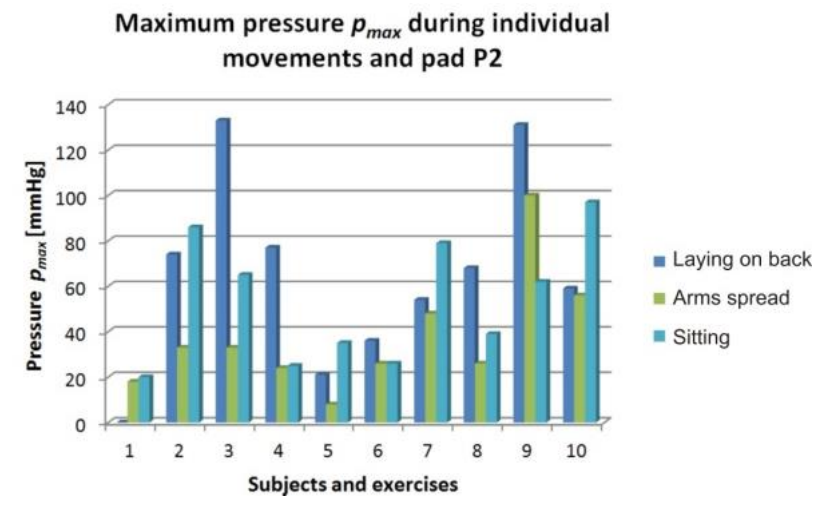

Fig. 11. Maximal contact pressure within the individual force pad (P2)

\section{Maximum area $S_{\max }$ during individual movements and pad P2}

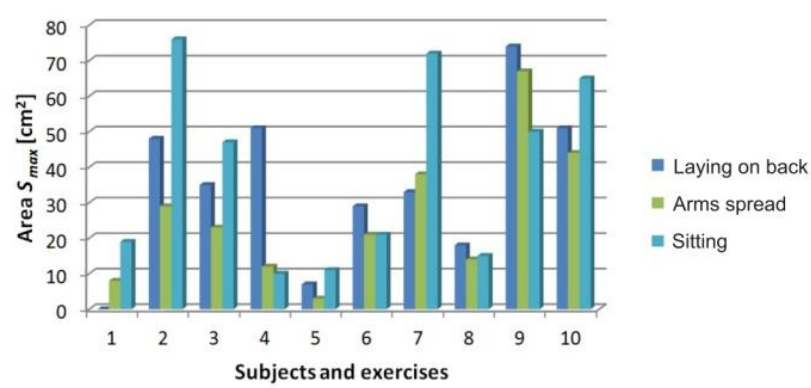

Fig. 12. Maximal contact surface within the individual movements (force pad P2)

If we eliminate extreme values out of the analysis, average pressures at three exercises on force pad $\mathrm{P} 2$ moved from approximately 0 to $80 \mathrm{mmHg}(0-10.6658 \mathrm{kPa})$. The average surfaces were also the largest while lying on the back, which is related to pressure at this position (movement). Maximal surfaces were higher also while sitting and leaning against a chair. 
Following the same method, outputs for standing position were obtained (Fig. 13). While standing without any other movement, sinusoid curves are visible and they represent increase and decrease in pressure at breathing. Within other movements, the output curves were so variable that it was not possible to evaluate them and clearly define reference process of neither pressures nor contact surfaces.

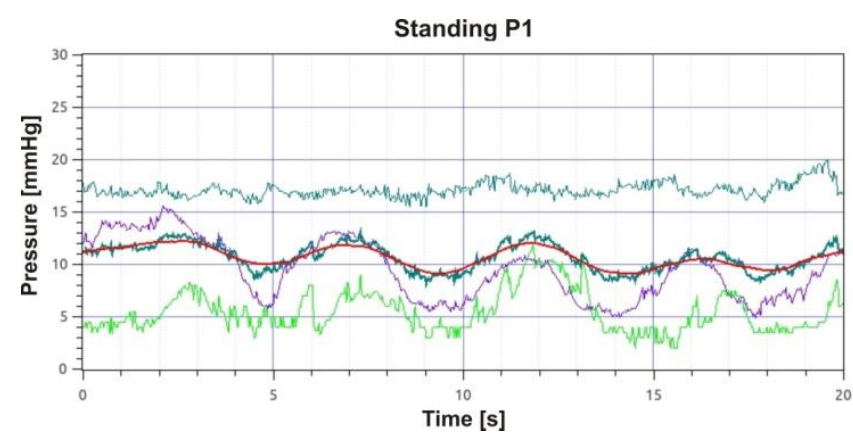

Fig. 13. Average pressures processes in time of standing position within all objects of measurement

\section{CONCLUSION}

Based on the analysis of the custom TACTILUS system, it was determined that the system from the point of view of range and sensitivity is correctly dimensioned. Chosen static and dynamic parameters were also tested. The overall measuring system uncertainty was obtained by the calculation from uncertainty A and uncertainty type $B$, where the value $u_{c}(p)=2.50 \mathrm{mmHg}$ was calculated.

Amplitude frequency noise spectrum does not contain any significant spectrum of line, it is flat and it responds to the thermal noise spectrum.

From the point of view of dynamic mat parameters, the calculations show that the time constant of transition to the second stable status is approximately 0.8 seconds. It means a relatively quick response to the weight change. This data will be verified based on the further measurements.

The aim of the analysis was also to create reference processes for all analyzed parameters and all implemented movements or exercises. In this case, reference curves were set up for kyphotisation and standing position. Regarding the other curves, it is necessary to carry out further large measurements and on their basis a database of reference curves of the most frequent movements or deliberately defined movements will be created. According to this database, clinical staff will be able to analyze pressures at measurements in ambulance regime (orthosis testing), but also in home regime where it will be clear which movements were done by the patient, if he applied the trunk orthosis correctly and which time period was the trunk orthosis used for.

\section{REFERENCES}

1. Bergeron Ch., Cheriet F., Ronsky J., Zernicke R., Labelle H. (2005), Prediction of anterior scoliotic spinal curve from trunk surface using support vector regression, Engineering Applications of Artificial Intelligence, 18, 973-983.

2. Berryman F., Pynsent P., Fairbank J., Disney S. (2008), A new system for measuring three-dimensional back shape in scoliosis, Eur Spine J., 17, 663-672.
3. Clin J., Aubin C. E., Parent S., Labelle H. (2010), A biomechanical study of the Charleston brace for the treatment of scoliosis, Spine, 1, 35(19), 940-7.

4. Gál, P., Toporcer, T., Vidinský, B., Hudák, R., Živčák, J., Sabo, J. (2009), Simple interrupted percutaneous suture versus intradermal running suture for wound tensile strength measurement in rats: $A$ technical note, European Surgical Research., Vol. 43, No. 1, 61-65.

5. Hermus J., Hulsbosch M., Guldemond N., Rhijn L. V. (2009), Developing a new brace with pressure measurements, 6th International Conference on Conservative Management of Spinal Deformities, Lyon, France, 1-1.

6. Hermus J., Monteban P., Guldemond N., Rhijn L. V. (2008), Pressure measurements in a new TLSO with a dynamic thoracic brace pad for idiopathic scoliosis, 5th International Conference on Conservative Management of Spinal Deformities, Athens, Greece, 1-1.

7. Krištof M., Hudák R., Takáčová A., Živčák J., Fialka L., Takáč R. (2010), Contact pressure measurement in trunk orthoses, IEEE International Joint Conferences on Computational Cybernetics and Technical Informatics, Timişoara, Romania, 175-179.

8. Kulesza E., Dąbrowski J. R., Sidun, J., Neyman A., Mizera J. (2012), Fretting wear of materials-Methodological aspects of research, Acta Mechanica et Automatica, 6(3), 58-61.

9. Penhaker M., Stankus M., Prauzek M., Adamec O., Peterek T., cerny M., Kasik V. (2012), Advanced Experimental Medical Diagnostic System Design and Realization, Electronics and Electrical Engineering, 117.1, 89-94.

10. Périé D., Aubin C. E., Lacroix M., Lafon Y. and Labelle H. (2004), Biomechanical modelling of orthotic treatment of the scoliotic spine including a detailed representation of the brace-torso interface, Medical and Biological Engineering and Computing, 42, 3, 339-344.

11. Praslicka D., Blažek J., Šmelko M., Hudák J., Čverha A., Mikita I., Varga R., Zhukov A. (2013), Possibilities of measuring stress and health monitoring in materials using contact-less sensor based on magnetic microwires, Magnetics, IEEE Transactions, 49.1, 128-131.

12. Skelly, A. (2007), The smart brace: Hi-tech scoliosis brace monitors pressure levels, patient compliance, Medical Post. [online], 43/25; 29.

13. Wong M. S., Mak A. F., Luk K. D., Evans J. H., Brown B. (2000), Effectiveness and biomechanics of spinal orthoses in the treatment of adolescent idiopathic scoliosis (AIS), Prosthet Orthot Int., 24(2):148-6.

14. Zach L., Horak L., Ruzicka P., Konvickova S. (2008), Knee Joint Edoprosthesis - Verification of Contact Pressures by Pressure Sensitive Films [online]., Sensor Products Inc.

15. Živčák J., Hudák R., Krištof M., Bednarčíková L. (2010), Surface pressure analysis in prosthetics and orthotics, Advances in Human Factors, Ergonomics, and Safety in Manufacturing and Service Industries, London, 845-854.

16. Živčák J., Krištof M., Hudák R. (2010), Pressure measurement in spinal brace - human body interaction area, Lékař a technika, 40/2, 20-24.

The work has been accomplished under the research project ITMS: 26220220185 MediPark Medical university science park in Kosice and the project ITMS No. 26220120060, Centre for research of control of technical, environmental and human risks for permanent development of production and products in mechanical engineering supported by the Research \& Development Operational Programme funded by the ERDF. 